\title{
Using Evolutionary Learning of Behavior to Find Weaknesses in Operating Systems
}

\author{
Jörg Denzinger and Tim Williams \\ Department of Computer Science, University of Calgary, \\ 2500 University Drive NW, Calgary, Alberta, T2N 1N4 Canada \\ \{denzinge,timw\}@cpsc.ucalgary.ca
}

\begin{abstract}
System security is an ongoing struggle between system designers and the hacking community. Human creativity within this community pushes software into areas never anticipated by the designers, thus revealing weaknesses. Evolutionary algorithms offer designers a new way to examine the viability of their code. Because of the use of randomness as well as direction based on evaluation, these algorithms help to simulate some aspects of the human creative process. In this work we show that already rather simple evolutionary searches allow us to find weaknesses in an operating system, a Linux version, resulting in a crash of the system and the necessity to reboot - a serious system flaw and security risk.
\end{abstract}

\section{Introduction}

In a world where more and more things are controlled by computers using more and more complex program systems, unanticipated behavior of these complex systems becomes more and more a problem (see [1]). The need to interact with human beings or other computer systems brings with it the danger of having to react to unforeseen situations, where the system behavior then becomes unpredictable, unreliable and potentially harmful (see [9]). System crashes due to "hostile" user behavior and viruses are examples of harmful interactions, where human beings use observations of a system, intuitive thinking and some basic knowledge about the system and its environment to wreak havoc around the world (see [2], [3], [10], [12] and for a more defensive approach [5]).

Naturally, testing and verification are intended to prevent coming into harmful situations within a system. But, in addition to never having enough resources, state-of-the-art testing methods do not really address the issue of unforeseen behavior of users or other systems, simply because they are unforeseen. The standard tests trying to cover the possible paths through program structures (see [11]) do not help a lot for programs that allow for input at every time and they do nothing about other systems, like the operating system or compilers, that the tested system relies on. Formal verification methods suffer from the same problems and additionally cannot cope with larger systems. Scenario based testing focuses on testing system behavior in its full context, but it requires someone envisioning the scenarios and then tests if the system reactions are adequate. It 
is not able to find scenarios that lead to unwanted behavior. In fact, in todays testing often there is nearly no focus on unwanted things, i.e. on trying to test for negative conditions.

If we look at how human users abuse systems by bringing them into unforeseen situations and taking advantage of the then "confused" system, then we can observe that a lot of human abilities come into play that are at the core of AI research: appropriate use of knowledge, learning from observations, dealing with not always predictable environments, and the use of intuition to suggest behavior. Consequently, AI techniques should play a key role in testing complex systems, but, so far, this is not the case.

In this paper, we present an on-line learning system, based on evolutionary techniques, that is used to test the Linux operating system (version 2.2.10) for user behavior that can crash the system. The basic idea of our EvoAttack system is to evolve sequences of operating system calls that are evaluated with regard to a given fitness function and that "breed" new sequences that hopefully achieve higher fitness levels. The best sequences are retained and re-evaluated in every generation of sequences. The computer system on which the sequences are evaluated is put under more and more "pressure" (relative to the fitness measure) until a sequence is produced that gets the target system to show a certain effect as the result of the cumulative efforts of all sequences it was exposed to.

The effect that our EvoAttack system wants to produce is a crash of the targeted system. Despite the fact that operating systems are very susceptible to timing issues and have a lot of safeguards to prevent user-level processes from harming the system kernel, EvoAttack was able to generate sequences of system call sequences that brought the target system into a state requiring a reboot in 70 percent of the "attack" attempts. And one of the fitness functions that were able to achieve this just measured the execution time of the system call sequences, a measure that can be easily obtained by outside observation.

\section{Some Linux Essentials}

The current three leading commercial operating systems all follow very different design philosophies which affect not only how they are written but how they function and how they are tested. The operating systems in question are:

- Linux: a monolithic kernel with loadable modules

- Microsoft Windows 2000: an object oriented kernel

- Apples OS-X which is a micro-kernel based on the Mach kernel

Each of these designs have large followings and different strengths and weaknesses. The kernel used for testing was a version of Linux (2.2.10), a reliable and well documented operating systems kernel. It is monolithic in nature, which means that the majority of the operating system resides as a single large block of code. This code is comprised of several major subsections, such as memory management, scheduling and resource management, which are eventually linked together to form the core or kernel of the operating system. Kernel developers 
User Programs

\begin{tabular}{|l|l|l|l|}
\hline \multicolumn{3}{|c|}{ Operating System Call Layer } \\
\hline \multirow{3}{*}{$\begin{array}{l}\text { File } \\
\text { System }\end{array}$} & Scheduler & Inter-process Communications & $\begin{array}{l}\text { Network } \\
\text { Protocol } \\
\text { Stack }\end{array}$ \\
\cline { 2 - 2 } & Memory Manager & Process Creation and Control & \\
\cline { 2 - 3 } & Signal Manager & Misc. System Resources & \\
\cline { 2 - 3 } & Virtual Memory Manager & \\
\hline \multicolumn{2}{|c|}{ Interupt Handlers and Drivers } \\
\hline \multicolumn{2}{|c|}{ hardware - memory, disk, network controller, clock, DMA, etc. } \\
\hline
\end{tabular}

Fig. 1. Basic Block Diagram of Linux Kernel.

Leffler, McKusick, Karels and Quarterman ([8]) in their work on the design of a version of UNIX called 4.3BSD, describe the functions supplied by their UNIX kernel. "The UNIX kernel provides four basic facilities: processes, a filesystem, communications, and system startup." The Linux kernel provides the same basic functionality. Figure 1 shows a very basic block diagram of this kernel.

There are really only two ways of accessing the kernel. The first method uses software interrupts and is known as the system call layer, this is the method accessible to common user programs. The second method uses hardware interrupts and allows access via the kernels driver level code. By only allowing access to the kernel through these two tightly controlled mechanisms a large number of potential security flaws are avoided. It is the software interrupt mechanism, typically called the operating systems call interface, that provides the starting point for many system attacks. Our research makes use of standard, legal system calls and therefore accesses the kernel, from user space, via software interrupts. Our method is equally applicable to other kernel access vectors such as the packets delivered via the Ethernet controller, thus opening the way to the testing of both protocol stack and web applications.

\section{Evolutionary On-Line Learning for Threat Testing}

The goal of threat based testing of a system is not to verify correct functionality, the assumption is that current "positive" test methods are sufficient for this level of testing. The threat testing methodology we propose here focuses on the idea of generating an interaction sequence with the system under test that puts this system into a state that fulfills some conditions(s) that are unwanted and/or intended to be normally unattainable, thus constituting a threat to the system.

To explain the general method, we assume that the system $\mathcal{S}$ to be tested accepts some interactions with the outside world that can be described as a 
sequence $c_{1}, \ldots, c_{m}$ of commands, out of a set $\mathcal{C}$ of possible commands, that achieve reactions in the system putting it into a sequence $s_{1}, \ldots, s_{m}$ of states. Note that we do not assume that $\mathcal{S}$ produces $s_{1}, \ldots, s_{m}$ solely based on $c_{1}, \ldots, c_{m}$. There might be other interactions with the system that we are not controlling; the system, for example, might be reacting to information about its environment gained by sensors outside of our control. As a consequence, the same sequence of commands might produce different sequences of states every time it is run.

From a security point of view, systems are at risk if there are command sequences that either always lead to a sequence of states that include a state fulfilling unwanted conditions or at least in more than a certain percentage of the command sequence executions. We assume that we are able to detect a system state that fulfills a certain unwanted condition, which means that there is a computable predicate $\mathcal{G}$ that applied to a state returns true, if the state fulfills the condition and else false.

We propose to use a Genetic Algorithm (GA, see [6] for an introduction) to produce command sequences that lead to states fulfilling $\mathcal{G}$. A GA works on a set of so-called individuals. Each individual is evaluated according to a so-called fitness function $f_{f i t}$ and new individuals are created by combining old individuals, selected based on their fitness but also including some random component, using so-called Genetic Operators. After a certain number of new individuals are generated, the same number of old indidviduals, usually the ones with the lowest fitness, are deleted to make room for the new individuals. The remaining old individuals together with the new ones constitute the new starting point for the next generation cycle.

This basic algorithm is well known and has many variants dealing with how the mixture of fitness value and randomness in the selection is achieved and what Genetic Operators are defined, for example. The key components to connect the general algorithm to the application for which it is used are how an individual is defined and how its $f_{f i t}$-value is computed. Naturally, the structure of an individual also influences what can be used as a Genetic Operator.

From the point of view of threat testing of a system $\mathcal{S}$, the obvious candidate for individuals are command sequences. The fitness value of an individual should indicate first, whether the sequence led to a state fulfilling $\mathcal{G}$ and if not, it should at least indicate how near the sequence came to such a state. Since we assume that $\mathcal{S}$ is not only dependent on the command sequence constituting an individual, the fitness should not be based on one evaluation by feeding the sequence to $\mathcal{S}$, but it should combine several such evaluation runs.

But for complex systems that, due to other influences on them, are rather unpredictable or even indeterministic, this approach to testing it with regard to $\mathcal{G}$ is too simple, resp. the search spaces the GA faces are too big and too volatile to be conquered by the testing GA. While the basic approach described above can also be seen as an off-line learning approach, we suggest to modify it to achieve an on-line learning threat testing system.

We still want to use sequences of commands as individuals, but we use sequences of a fixed length $k$ and $k$ is not very large. We also still propose to 
evaluate the individuals by sending them as command sequence to $\mathcal{S}$ and the fitness measure is still how near executing the sequence came to achieve $\mathcal{G}$. But this fitness measure is not solely based on the sequence that was just executed, it is based on all sequences that were evaluated before as well as on all other outside influences that occured since the system $\mathcal{S}$ was in use. So, the whole unpredicatability of the system $\mathcal{S}$ is not seen as a liability anymore, we use it to our advantage. This means that the fitness of an individual depends on when we do the evaluation and therefore we repeat the evaluation of all individuals in a generation every time we start the repetition of the basic loop of the GA. Thus individuals that seem to bring the system nearer to a state fulfilling $\mathcal{G}$ at one point in time either add to the pressure towards $\mathcal{G}$ or they will be discarded later. Hence we have an on-line learning system.

The fitness function depends a lot on the tested system $\mathcal{S}$ and the condition $\mathcal{G}$. But the possible Genetic Operators can be easily defined in general. If we have two command sequences $c_{1}, \ldots, c_{k}$ and $c_{1}^{\prime}, \ldots, c_{k}^{\prime}$, then Crossover randomly selects an $i$ with $1 \leq i \leq k$ and the resulting sequence is $c_{1}, \ldots, c_{i}, c_{i+1}^{\prime}, \ldots, c_{k}^{\prime}$. To perform a Mutation on $c_{1}, \ldots, c_{k}$, we again select a random $i$ and a command $\mathrm{c} \in \mathcal{C}$ with $\mathrm{c} \neq c_{i}$ and the new individual is $c_{1}, \ldots, c_{i-1}, \mathrm{c}, c_{i+1}, \ldots c_{k}$.

While this concept of threat testing was inspired by works on learning behavior for agents, like [4], this kind of usage of these methods is new. [11] suggested the usage of evolutionary methods to develop tests covering the branches in programs controlling embedded systems. But these systems do not exhibit at all the level of undeterministic behavior we are dealing with and they are far away from the complexity of the systems we are targeting. Also, knowledge about the program structure is necessary for Wegener's approach to work, whereas our method does not depend on the program structure at all. [7] also uses evolutionary methods to evolve new tests for a controller, but out of a set of known faults of this controller. Obviously, this is mainly a recombination effort, not the construction of a new threat, as in our approach.

\section{Crashing Linux}

Our testing methodology focuses on the idea of generating, within the operating system, a variety of states that are unwanted and intended to be normally unattainable. In this case the goal was to drive the operating system into an unusable state. A typical experimental run ranged in duration from 2 to 3.5 days which limited the actual number of runs included in our evaluation. From a security point of view, the results are, however, significant (over 70 percent success rate), since essentially already one successful run could cause a lot of damage.

We used a fully instrumented Linux (version 2.2.10) kernel for these experiments. This version of the kernel, although older, is stable, familar and well documented. There are a few documented flaws of this kernel version, which was one of our reasons for choosing it, since initially we wanted to target these flaws with our EvoAttack system. Naturally, after the initial experiments that found 


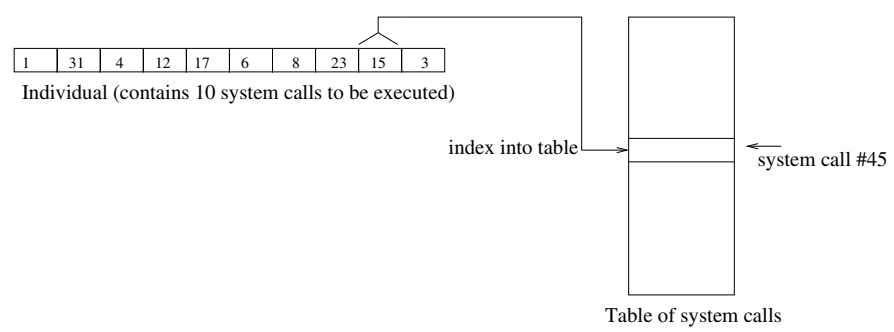

Fig. 2. Example of gene to actual call mapping.

different, unknown problems, we concentrated on evaluating these new problems. A second reason was that revealing new flaws would not compromise the currently deployed systems. Most routines were compiled with the gcc instrumenting option and a trace routine was provided to log when a kernel routine was entered or exited. A custom driver was written to allow access to the kernel trace buffer created via the instrumentation routines. The behaviour which can be monitored easily consists of kernel execution times and internal kernel execution paths. The entrance and exit times of each kernel routine are monitored and the execution of each system call is traced. System memory usage was not monitored due to the already large size of the test data structures. The data captured allows for the analysis of many values such as:

- total call time

- time spent in certain kernel areas or routines

- internal execution path, entry into error processing routines

- variations in time or path execution from a statistical normal

An individual, within the EvoAttack system, is comprised of a sequence of valid operating system calls. Not all of the kernels system calls were part of the acceptable list since some calls, such as kill, would cause an early termination of the individual. Therefore each element of the call sequence is an index into an array of possible system calls and not the actual call number as pushed onto the stack and seen by the kernel (see Figure 2). Most of the operating system calls require parameter values. While we think that these can also be evolved, for our experiments we used fixed values for each call. The selected values are legitimate values for the particular call and do not cause any known harm.

The implementation of our test system involved the use of two separate computers. The target system, which ran the actual individuals and performed the kernel tracing, communicated with a host system via the parallel link. The host system implemented the GA and calculated the fitness functions. The isolation of the target was required to minimize the execution of system calls that were not directly related to the tested individuals. The communications link was controlled by direct access to the parallel port and not by the execution of any related system calls. The kernel trace buffer was accessed from user space via a custom driver written for this purpose. It should be noted that the target system's performance is significantly less than that of the host. This was done for 
two reasons: cost and timing. The first reason is self explanatory but the rational for timing needs to be examined. Some of the interrupts generated by a system are not directly influenced by the speed of the processor. For example, the system clock generates ticks at the same rate regardless of the processor clock rate. This means that the ratio of interrupts serviced vs kernel code executed will be much higher on lower speed systems. This allows less chance for the kernel to recover from interrupt related problems.

The host randomly creates the initial generation of individuals. An individual is sent to the target via the parallel link. Execution of each individual is done by creating a user level task, starting the trace, executing the individual's call sequence, terminating the trace and then allowing the task to terminate. The trace is then transmitted to the host for analysis.

In order to implement threat testing, we needed a fitness function. Since the actual target state or error is not known before testing, the initial search space is large. To help with the exploitation of the search space, certain kernel stress conditions, such as increased activity and/or execution time within the kernel are used as fitness functions. EvoAttack was quite successful using only these two, rather primitive, fitness functions. The first, $f_{\text {fit,time }}$, was a measure of the time spent in execution for each individual (a function which does not require kernel tracing to measure). The second fitness function, $f_{\text {fit,call }}$, was a measure of the number of kernel routines used by an individual during execution. This function made heavy use of the kernel trace facilities that we had provided. In keeping with our need to isolate the target system both functions were calculated by the host based on the trace information provided by the target.

The initial population were 100 random individuals (produced using 32 different system calls). Each individual contains a sequence of 10 system calls. Our GA then used a simple elitist selection method for the selection of parents and the creation of the next generation. All of the individuals of the generation are ranked, based on their fitness value. The lowest 12 percent of the population are removed and the top 24 percent of the population is allowed to breed. New individuals are created via the usual random point crossover. All of the resulting population is then considered for exposure (based on a programmable mutation rate) to a single point mutation operator. This is realized by either incrementing or decrementing the value representing the call. The choice of increment of decrement is random.

Each individual in the new generation is executed and a new fitness value calculated. Note that this value is dependent on previously evaluated individuals! It is useful to think of a kernel as a state machine whose operation is affected, not only by inputs and previous states, but also by time. Events, both random and periodic (typically hardware interrupts), cause the system to jump into specific interrupt service routines. These routines access and modify kernel data structures. When a data structure is modified by the interrupt handler, it could occur at a time when another section of the kernel had partly completed a modification to the same data structure. This sort of race condition is commonly faced by most shared memory systems. The kernel uses flags and interrupt masks to min- 
Table 1. Evaluation results with different fitness functions.

\begin{tabular}{|l|c|c||c|c|}
\hline Fitness function & Runs & Mut. Rate & Success Rate & Observed Error Types \\
\hline \hline$f_{\text {fit,time }}$ & 8 & $75 \%$ & $57.1 \%$ & $1,2,3$ \\
\hline$f_{\text {fit,time }}$ & 5 & $65 \%$ & $60.0 \%$ & $1,2,3$ \\
\hline$f_{\text {fit,time }}$ & 7 & $40 \%$ & $71.4 \%$ & $1,2,3$ \\
\hline$f_{\text {fit,call }}$ & 10 & $65 \%$ & $70.0 \%$ & 2,3 \\
\hline
\end{tabular}

imize the possiblity of data corruption. The main problem with this situation, as it pertains to testing, is that an interrupt can change the operating systems internal state and therefore the execution path taken by the kernel's code. For example, if an interrupt affects a flag, which is then tested and acted on by the kernel, the outcome will be different if the flag is tested before or after the interrupt changes it. A microsecond's difference in interrupt arrival time between two test runs can cause significantly different operating systems states.

\section{Experimental Evaluation}

For our experiments, we tested a machine that was only running the individuals for evaluation; no other user processes were active on the target. So, problems due to the interaction of several user processes are outside of the scope of our experiments. As mentioned earlier, it was anticipated that the final fitness functions would be very complex, however the two most primitive functions proved to be more than enough to provide our proof-of-concept.

Using the total execution time of the individual as the first fitness function $f_{\text {fit,time }}$ proved to be successful (see Table 1 ). The crash rate was dependent on the mutation rate ranging from 40 to 75 percent. As was expected, the runs with somewhat lower mutation rates yielded better results, due to a greater exploitation of the results of individuals and less exploration of new sequences. The error conditions experienced by the test system consisted of three types:

1. the inability for root (or any user) to log into the system. Although the login prompt accepted input, the password prompt would never appear.

2. the inability to execute most programs. The system would generate a segmentation fault (usually indicative of a memory/pointer problem) upon the attempted execution of most commands. The system memory utilization did not appear to rise and fall substantially and no swap memory was in use.

3 . the target program would stop communications, this error was always observed in conjunction with one of the other error conditions.

All of these faults could be related to issues within the memory management subsystem of the kernel.

The second fitness function $f_{\text {fit,call }}$ was simply the total number of calls to kernel routines during the execution of the individual. As a fitness function, this yielded even better results than the use of execution times (see Table 1), perhaps due to the increased error recovery and cleanup which can be complex, 
Table 2. Subsequences for $f_{f i t, \text { call }}$ shortly before a crash.

\begin{tabular}{|l||c|c|}
\hline Subsequence & In top 24 Individuals & In all Individuals \\
\hline $29,13,13,25,31,29,1$ & $57.9 \%$ & $19.8 \%$ \\
\hline $29,13,13,25,31,29$ & $57.9 \%$ & $20.9 \%$ \\
\hline $29,13,13,25,31$ & $57.9 \%$ & $21.5 \%$ \\
\hline $29,13,13,25$ & $68.4 \%$ & $26.9 \%$ \\
\hline $29,13,13$ & $68.4 \%$ & $27.2 \%$ \\
\hline 29,13 & $78.9 \%$ & $32.3 \%$ \\
\hline
\end{tabular}

but potentially fast, activities. All errors were of the second and third type described above (segmentation fault and communications error).

Using the second fitness function, distinct subsequences were observed by the end of the test. The top 24 individuals (after ranking) are the ones used in the creation of new individuals so it is their sequences which will be reinforced in the new generation. Table 2 presents an analysis of the subsequences of system calls observed in the last generation of a successful test where the target system crashed during the execution of the 20th individual in generation 590 . We observed rather long subsequences - which would be called building blocks in evolutionary computation. For example, more than 50 percent of the top 24 percent individuals included the subsequence $29,13,13,25,31,29,1$ that was also in nearly 20 percent of all individuals generated in this run. Among the longest sequences occuring in more than one individual was $29,13,13,25,31,29,1,27,16$, which maps to the following $\mathrm{C}$ system calls:

29: pipe(Tpfd);

13: stat (Tname, (struct stat $*$ ) Tbuf);

13: stat (Tname, (struct stat $*$ ) Tbuf);

25: rename (Tnewname, "testname3");

31: setgid(Mygid);
29: pipe(Tpfd);

1: $\operatorname{read}(\mathrm{Tfd}$, Tbuf, Tcnt);

27: rmdir ("testdir");

16: setuid (Myuid);

This represents a sequence of system calls which is very unusual and not likely to occur in normal user programs.

All in all, our experiments show that our method of threat testing can reveal weaknesses in operating systems. Since operating systems are among the very complex systems and very difficult to test with regard to unwanted behavior, we see our EvoAttack system as proof that using AI can improve testing, and as a consequence system security and reliability, a lot. Threat based testing can be a very valuable addition to the testers' arsenal of tools, since it covers areas not examined well by other mechanisms. Threat testing should not be used alone since there is naturally no guarantee that 10 runs without a crash mean that a system has no problems.

\section{Conclusion and Future Work}

We presented an AI-based method for testing complex systems with regard to the possibility that interactions with the system can threaten it, resulting in 
unwanted or even dangerous behavior. In our proof of concept, our threat testing of a stable version of the Linux operating system revealed the possibility to crash the system from a normal user account. This was achieved with either of two rather primitive fitness functions that guided the evolutionary on-line learning that is the core of our method. One of these functions only measured the execution time of the interaction commands, a measure that can be easily taken without any additional specialized access to the system. The reveiled problem has to be considered a serious flaw of the tested system that was only revealed by our threat testing method.

As stated above, so far we consider our research into this topic only as a proof of concept. There are many possible improvement and additional goals that have to be addressed in the future. In addition to applying the general method of threat testing to additional applications (starting with more current versions of Linux, different operating systems, and other kinds of complex program systems, like web servers or data base systems), we expect the development of fitness functions measuring different kinds of threats an important area. We did not expect that the simple fitness functions we tried first, to get a feeling of what happens, would already be successful. With the use of threat testing by system developers, many systems should not be susceptible to such rather simple threats in the future. But there will be more intelligent threats and consequently the fitness functions will have to incorporate more knowledge to test for such threats.

\section{References}

1. J. Branke. Evolutionary Optimization in Dynamic Environments, Kluwer, 2002.

2. D. Boneh and D. Brumley. Remote timing attacks are practical, Proceedings of the 12th Usenix Security Symposium, 2003, pp. 1-14.

3. D.E. Denning. Information Warfare and Security, Addison-Wesley, 1999.

4. J. Denzinger and M. Kordt. Evolutionary On-line Learning of Cooperative Behavior with Situation-Action-Pairs, Proc. ICMAS-2000, IEEE, 2000, pp. 103-110.

5. S. Hofmeyr and S. Forrest. Architecture for an artificial immune system, Evolutionary Computation Journal, 8(4), 2000, pp. 443-473.

6. D.E. Goldberg. The Design of Innovation: Lessons from and for Competent Genetic Algorithms, Kluwer, 2002.

7. A.C. Schultz, J.J. Grefenstette and K.A. De Jong. Adaptive Testing of Controllers for Autonomous Vehicles, Proc. Symposium on Autonomous Underwater Vehicle Technology, IEEE, 1992, pp. 158-164.

8. S.J. Leffler, M.K. McKusick, M.J. Karels, and J.S. Quarterman. The Design and Implementation of the 4.3BSD UNIX Operating System, Addison-Wesley, 1989.

9. G. Thaller. Software Engineering for Real-time and Embedded Systems, BHVVerlag, 1997.

10. United States Army. Army Field Manual- F100-6: Information Operations, 1996.

11. J. Wegener. Evolutionary testing of embedded systems, In Evolutionary Algorithms for Embedded Systems Design, Kluwer, 2003, pp. 1-34.

12. The WildList Organization International. PC viruses in-the-wild - real-time, http://www.wildlist.org/WildList/Real-Time.htm, as seen Oct 2003. 